\title{
Underwater cold forceps polypectomy for an adenoma within a cecal diverticulum o
}

\author{
Sho Sasaki ${ }^{1}$, Jun Nishikawa ${ }^{2}$, Isao Sakaida ${ }^{3}$ \\ ${ }^{1}$ Department of Gastroenterology, Saiseikai Yamaguchi Hospital, Yamaguchi; ${ }^{2}$ Faculty of Laboratory Science, Yamaguchi University Graduate School of \\ Medicine, Ube; ${ }^{3}$ Department of Gastroenterology and Hepatology, Yamaguchi University Graduate School of Medicine, Ube, Japan
}

Endoscopic resection of a polyp involving a colonic diverticulum is challenging. As there is no muscularis propria within a diverticulum, the potential risk of perforation associated with endoscopic resection must be considered. ${ }^{1,2}$

A 72-year-old male patient underwent follow-up colonoscopy after surgery for rectal cancer. A sessile polyp, $3 \mathrm{~mm}$ in size, was found in a cecal diverticulum. The underwater method eliminated halation and expanded the diverticulum cavity, making it possible to observe the lesion stably (Fig. 1A, Supplementary Video 1). Subsequently, underwater cold forceps polypectomy (CFP) was performed using Radial Jaw4-Jumbo cold polypectomy forceps (Boston Scientific, Tokyo, Japan) (Fig. 1B, C). Observation using narrow-band imaging and continuous irrigation confirmed that there were no residual lesions or perforations (Fig. 1D), and the wound was closed using clips. The pathological result was a tubular adenoma (Fig. 2).

The underwater method made it possible to expand the diverticulum cavity and float the lesion, allowing CFP to be performed safely. ${ }^{3}$ Cold biopsy forceps polypectomy was performed to avoid the risk of delayed perforation associated with

\footnotetext{
Received: December 30, 2020 Revised: January 13, 2021

Accepted: January 14, 2021

Correspondence: Sho Sasaki

Department of Gastroenterology, Saiseikai Yamaguchi Hospital, Midorimachi 2-11, Yamaguchi 753-8517, Japan

E-mail: sho.ssk.nv21@gmail.com

(a) This is an Open Access article distributed under the terms of the Creative Commons Attribution Non-Commercial License (http://creativecommons.org/licenses/by-nc/4.0/) which permits unrestricted non-commercial use, distribution, and reproduction in any medium, provided the original work is properly cited.
}

hot polypectomy. ${ }^{4}$ This technique may be useful for cold snare polypectomy of larger polyps extending into a diverticulum. ${ }^{5}$ However, it would not be safe for large polyps involving more than one-fourth of a diverticulum. To the best of our knowledge, this is the first reported case of underwater CFP for an adenoma within a colonic diverticulum.

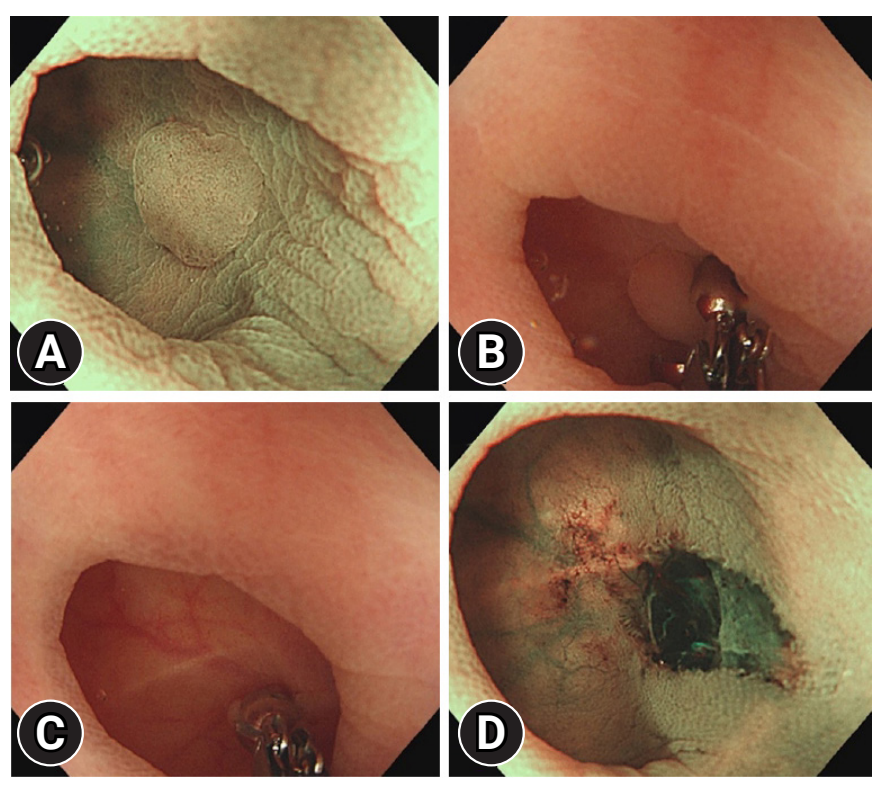

Fig. 1. Underwater cold forceps polypectomy. (A) A sessile polyp $3 \mathrm{~mm}$ in size was found in a cecal diverticulum. The underwater method improved polyp visualization. Narrow-band imaging (NBI). (B, C) Underwater cold forceps polypectomy was performed. (D) Observation using NBI and continuous irrigation confirmed that there were no residual lesions or perforation. 


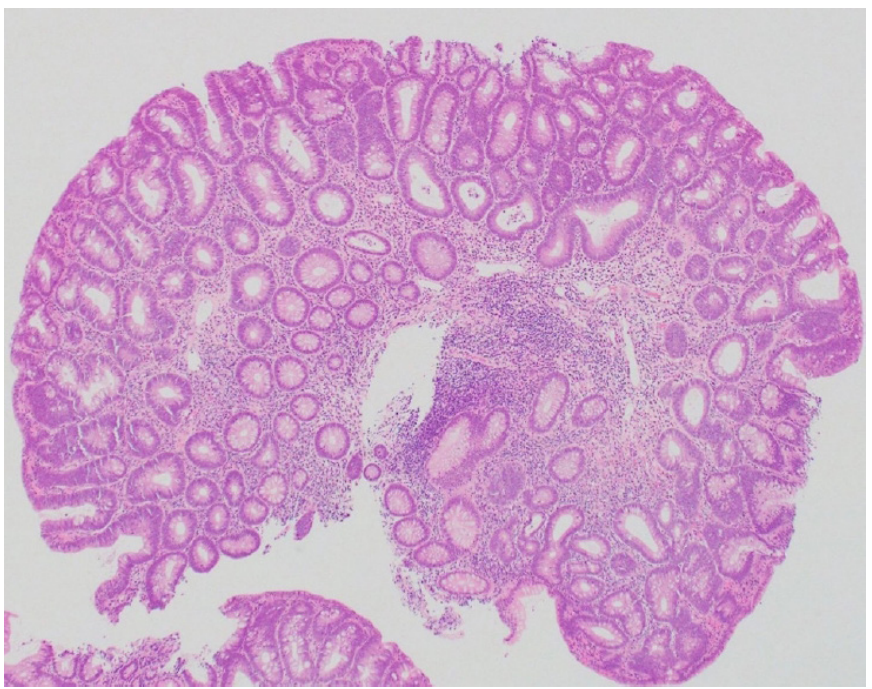

Fig. 2. Resected specimen (hematoxylin \& eosin, $\times 10$ ). Histopathological examination revealed a tubular adenoma.

\section{Supplementary Material}

Supplementary Video 1. Underwater cold forceps polypectomy for an adenoma within a cecal diverticulum (https://doi.org/ 10.5946/ce.2021.038.v001).

Supplementary materials related to this article can be found online at https://doi.org/10.5946/ce.2021.038.

\section{Conflicts of Interest}

The authors have no potential conflicts of interest.

\section{Funding}

None.

\section{Acknowledgments}

We express our gratitude to Dr. Shuji Wasaki (Wasaki Clinic, Tsuwano, Japan) for his careful endoscopy and detection of the lesion.

\section{Author Contributions}

Conceptualization: SS; Project administration: SS, JN; Supervision: IS; Writing-original draft: SS; Writing-review \& editing: SS, JN, IS.

\section{ORCID}

Sho Sasaki

https://orcid.org/0000-0003-4043-5562

Jun Nishikawa

https://orcid.org/0000-0002-6695-9754

Isao Sakaida

\section{REFERENCES}

1. Valli PV, Kaufmann M, Vrugt B, et al. Endoscopic resection of a diverticulum-arisen colonic adenoma using a full-thickness resection device. Gastroenterology 2014;147:969-971.

2. Carmo J, Marques S, Chapim I, et al. Elastic band ligation for the removal of a colonic tubular adenoma in a diverticulum. Endoscopy 2015;47 Suppl 1:E490-E491.

3. Binmoeller KF, Weilert F, Shah J, et al. "Underwater" EMR without submucosal injection for large sessile colorectal polyps (with video). Gastrointest Endosc 2012;75:1086-1091.

4. Kubosawa Y, Nishizawa T, Kinoshita S, et al. Cold snare polypectomy for polyp adjacent to colonic diverticulum. VideoGIE 2018;3:85-86.

5. Yanagisawa F, Inoki K, Katagiri A, et al. Cold snare polypectomy in combination with a traction device for resection of a colonic adenoma partially extending into a diverticulum. VideoGIE 2020;5:580582. 